\title{
A WAVELET BASED LIKELIHOOD RATIO TEST FOR THE HOMOGENEITY OF POISSON PROCESSES
}

\author{
Y. Taleb and E. A. K. Cohen \\ Department of Mathematics, Imperial College London, \\ South Kensington Campus, London SW7 2AZ, United Kingdom
}

\begin{abstract}
Estimating the rate (first-order intensity) of a point process is a task of great interest in the understanding of its nature. In this work we first address the estimation of the rate of an orderly point process on the real line using a multiresolution wavelet expansion approach. Implementing Haar wavelets, we find that in the case of a Poisson process the piecewise constant wavelet estimator of the rate has a scaled Poisson distribution. We apply this result in the design of a likelihood ratio test for a multiresolution formulation of the homogeneity of a Poisson process. We demonstrate this method with simulations and provide Type 1 error and empirical power plots under specific models.
\end{abstract}

Index Terms - Point processes, rate estimation, wavelet analysis, hypothesis testing

\section{INTRODUCTION}

The use of point processes to model random events in time and space is ubiquitous across science and engineering. A temporal point process can be represented at time $t \in \mathbb{R}$ as $N(t)$, which is a random integer indicating the number of events to have occured in the time-interval $(0, t]$. Point processes are often described using functions known as the first-order intensity (also called rate or intensity function) and the second-order intensity. These intensities characterize a point process and are directly related to properties such as homogeneity and stationarity. Since the estimation model in [1] for the general but limited class of stationary point processes, the challenge of estimating the rate of point processes has been treated from various point of views, with either parametric (e.g. [2]) or non-parametric methods. A class of non-parametric methods for the estimation of the rate of multivariate point processes is discussed in [3] from a multiplicative intensity model, with kernel smoothing applied later in this particular formulation [4].

In this paper we choose to work with wavelet expansions of the rate, which belong to the non-parametric class of estimation methods. Wavelet functions serve as bases of the space of square-integrable functions $L^{2}(\mathbb{R})$. There has been some interest in applying wavelets on point processes, as in [5] where wavelet coherence is applied on point processes over the real line. Our work finds its origins in the framework introduced in [6], where a wavelet decomposition of the rate and second-order intensity is proposed. As far as the rate is concern, a much clearer formulation for its wavelet estimator is derived in [7], with expressions of the expectation and the variance of this estimator provided for general point processes and wavelet families. Further to this, the estimation method presented in [8] is based on the shrinkage of Haar wavelet coefficients of a discretization of burst-like Poisson processes without any preprocessing. However, these papers lack distributional results which are required for inference or extended statistical analysis.

In this paper, we derive the distribution of a Haar wavelet expansion estimator of the rate function under a general Poisson process model. From this, we then go on to design a likelihood ratio test for a multiresolution formulation of the homogeneity of a Poisson process from a set of local rate estimates. Testing the homogeneity of a Poisson process has been a widely addressed task. The reader is directed to [9] for a comparative power study of a large variety of tests for a constant rate against an increasing trend, [10] and [11] for examples of score statistics and [12] for recent non-parametric procedures. Also, some tests for complete spatial randomness can be narrowed to a single dimension, i.e. [13]. Homogeneity is a very strong property, and for this reason we use a multiresolution view of homogeneity to define and test a property which we call $J^{t h}$-level homogeneity. A similar likelihood ratio test has been proposed in [14] for the piecewise homogeneity of a Poisson process, where the rate is analysed on subintervals of the trajectory. The authors notably establish distribution results of their test statistic under a specific alternative model. However, no scheme was proposed for the choice of these subintervals. We demonstrate that an approach based on the Haar wavelet multiresolution analysis provides an elegant way of naturally defining them.

This work is supported in part of the EPSRC 


\section{POINT PROCESSES ON THE REAL LINE}

A one-dimensional point process $N$ can be viewed as a counting process, with $N(a, b)$ being the number of events in any interval of the form $(a, b]$. We will specifically use the notation $N(t)$ for the interval $(0, t]$. We can sometimes encounter the notion of simple point process, that means a point process whose realizations contain no coincident points. Literature generally refers to a similar mathematical characterization known as orderliness [15]. A point process $N$ on $\mathbb{R}$ is called orderly if for all $t \in \mathbb{R}, N(\{t\}) \in\{0,1\}$ almost surely. For a comprehensive overview of the theory of point processes see [16].

The differential process is defined as $\mathrm{d} N(t)=N(t+\mathrm{d} t)-N(t)$. In the case of an orderly point process, $\mathrm{d} N(t) \in\{0,1\}$. The differential process provides us with the two following results :

$$
E\left\{\int \varphi(t) \mathrm{d} N(t)\right\}=\int \varphi(t) E\{\mathrm{~d} N(t)\}
$$

for all bounded measurable function $\varphi$ with compact support, and $E\{\mathrm{~d} N(t)\}=\mathrm{d} E\{N(t)\}$. Further to this the stochastic integral is given as

$$
\int \varphi(t) \mathrm{d} N(t)=\sum_{i} \varphi\left(\tau_{i}\right)
$$

where $\tau_{i}$ are the event times of the process. These results will prove very useful when deriving the estimator for the first-order intensity.

Our object of interest is the first-order intensity or rate. It is defined as

$$
\lambda(t)=\frac{E\{\mathrm{~d} N(t)\}}{\mathrm{d} t}
$$

or equivalently $\lambda(t) \mathrm{d} t=\mathrm{P}(\mathrm{d} N(t)=1)$ The reader is directed to [7] and [15] for further details and properties of the rate.

\subsection{Poisson Process}

Let $\mathbb{X}$ be a subset of $\mathbb{R}$ and $\mathcal{X}$ its associated Borel $\sigma$ algebra. Let $\mu$ be measure on $\mathbb{X}$ with $\mu(K)<\infty$ for any compact set $K \in \mathcal{X}$. Then $N$ is an (inhomogeneous) Poisson process with mean measure $\mu$ if :

- $\forall B \in \mathcal{X}, \mathrm{P}(N(B) \in\{0,1, \ldots\})=1$, and for any collection of disjoint sets $B_{1}, B_{2}, \ldots, B_{k} \in \mathcal{X}$ the random variables $N\left(B_{1}\right), N\left(B_{2}\right), \ldots, N\left(B_{k}\right)$ are independent.

- $\forall s \in \mathbb{X}$,

$$
\begin{aligned}
& \mathrm{P}(N(\mathrm{~d} s)=0)=1-\mu(\mathrm{d} s)+o(\mu(\mathrm{d} s)) \\
& \mathrm{P}(N(\mathrm{~d} s)=1)=\mu(\mathrm{d} s)+o(\mu(\mathrm{d} s)) \\
& \mathrm{P}(N(\mathrm{~d} s)>1)=o(\mu(\mathrm{d} s))
\end{aligned}
$$

where $\mathrm{d} s$ is an infinitesimal region located at $s$.

These properties lead to the conclusion that $N(B)$ has a Poisson distribution with mean $\mu(B)$. In dimension one, $\mu(B)=$ $\int_{B} \lambda(t) \mathrm{d} t$. A homogeneous Poisson process is an Poisson process where for all $B \in \mathcal{X}, \mu(B)=\lambda \nu(B)$ with $\lambda>0$ and $\nu$ is the Lebesgue measure on $\mathbb{X}$. Its rate is thus a constant function.

\section{WAVELET BASED ESTIMATION OF THE RATE}

Let us consider the following wavelet expansion for the rate function $\lambda$ on a time interval of the form $[0, T]$ :

$$
\lambda(t)=\sum_{k \in \mathbb{Z}} \delta_{j_{0}, k} \phi_{j_{0}, k}(t)+\sum_{j \in j_{0} \mathbb{Z}} \sum_{k \in \mathbb{Z}} \beta_{j, k} \psi_{j, k}(t)
$$

where $j_{0} \mathbb{Z}=\left\{z \in \mathbb{Z}: z \geq j_{0}\right\}$ and $j_{0}$ is a fixed integer. The functions $\phi_{j_{0}, k}$ and $\psi_{j, k}$ are respectively the father and mother wavelet functions. These families are built from the scheme $\psi_{j, k}(t)=2^{j / 2} \psi\left(2^{j} t-k\right)$. As shown in [17], the collection of functions $\left\{\psi_{j, k},(j, k) \in \mathbb{Z}^{2}\right\}$, is an orthonormal basis of $L^{2}(\mathbb{R})$. Using (2), the wavelet coefficients are estimated as following:

$$
\begin{gathered}
\hat{\delta}_{j_{0}, k}=\int_{0}^{T} \phi_{j_{0}, k}(t) \mathrm{d} N(t)=\sum_{\tau_{i}} \phi_{j_{0}, k}\left(\tau_{i}\right) \\
\hat{\beta}_{j, k}=\int_{0}^{T} \psi_{j, k}(t) \mathrm{d} N(t)=\sum_{\tau_{i}} \psi_{j, k}\left(\tau_{i}\right) .
\end{gathered}
$$




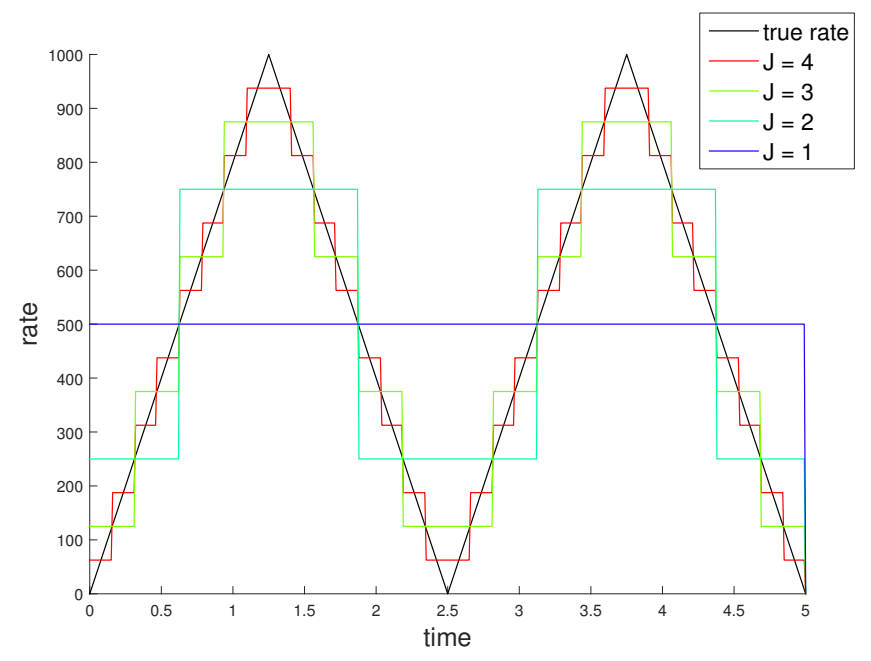

Fig. 1. Wavelet reconstruction of a piecewise triangular rate for different maximum resolutions: $J \in\{1,2,3,4\}$. The wavelet reconstructions at $J=0$ and $J=1$ are equal.

Equations (1) and (3) show $\hat{\delta}_{j_{0}, k}$ and $\hat{\beta}_{j, k}$ are unbiased estimators of the true wavelets coefficients of the rate, i.e.

$$
\begin{aligned}
& E\left\{\hat{\delta}_{j_{0}, k}\right\}=\int_{0}^{T} \phi_{j_{0}, k}(t) E\{\mathrm{~d} N(t)\}=\int_{0}^{T} \phi_{j_{0}, k}(t) \lambda(t) \mathrm{d} t \\
& E\left\{\hat{\beta}_{j, k}\right\}=\int_{0}^{T} \psi_{j, k}(t) E\{\mathrm{~d} N(t)\}=\int_{0}^{T} \psi_{j, k}(t) \lambda(t) \mathrm{d} t .
\end{aligned}
$$

In practice we construct a wavelet expansion of a function up to some finite scale $J$ instead of using the full wavelet basis. Considering the wavelet expansion (4) at different scales provides a multiresolution analysis of the rate function. With a simplification of the indices for wavelets with compact support and a restriction to $[0, T]$, we define the unbiased estimator of the rate function:

$$
\hat{\lambda}^{J}(t)=\sum_{k=0}^{2^{j_{0}}-1} \hat{\delta}_{j_{0}, k} \phi_{j_{0}, k}(t)+\sum_{j=j_{0}}^{J} \sum_{k=0}^{2^{j}-1} \hat{\beta}_{j, k} \psi_{j, k}(t)
$$

where $J$ is the highest level of resolution and $j_{0}$ the coarsest level of resolution. These levels indicate the scale of the analysis we are performing on point process data. In practice we will always set $j_{0}=0$ and the maximum level of resolution $J$ to a value that is of interest, or a compromise between precision of analysis and computing time. In [7] they show that under some common regularity assumptions, the rate estimate is asymptotically unbiased as $J \rightarrow \infty$.

\subsection{Statistical Analysis}

The wavelet basis used in our analysis will be the Haar basis (rescaled if $\mathrm{T}$ is different than 1), primarily because it lends itself to a rigorous statistical analysis. We define a subdivision $S_{J}=\left\{s_{i}\right\}_{i=0}^{2^{J+1}-1}$ of the interval $[0, T]$ based on the highest level of resolution $J$. The elements of this subdivision, $s_{i}=\left[T \frac{i}{2^{J+1}}, T \frac{i+1}{2^{J+1}}\right)$, are all the half-supports of the Haar wavelets at scale $J$ and form disjoint $2^{J}$ sub-intervals of $[0, T]$. The choice of Haar wavelets is in part motivated by the fact that the wavelet reconstruction of the rate is a piecewise constant function, and hence we can define a wavelet reconstruction vector $\left(\lambda_{0}^{J}, \cdots, \lambda_{i}^{J}, \cdots, \lambda_{2 J+1}^{J}-1\right)$ where $\lambda_{i}^{J}$ is the wavelet reconstruction of the rate on the subinterval $s_{i}$. We use this formulation to introduce a property we call $J$ th-level homogeneity:

Definition 3.1 A Poisson process with rate $\lambda(t)$ is considered level $J$ homogeneous if the Haar wavelet reconstruction of the rate at resolution $J$ is constant for all $t$. That is, $\lambda_{0}^{J}=\lambda_{1}^{J}=\ldots=\lambda_{2^{J+1}-1}^{J}$.

It can be easily proved that a level $J$ homogeneous Poisson process also satisfies this condition for all resolutions $0 \leq j \leq J$ [18]. The concept of $J$ th level homogeneity goes side by side with the idea of a multiresolution analysis of the intensity function and provides a natural way of studying on what scales the rate function exhibits variability. We also introduce the following property: 
Definition 3.2 A Poisson process with rate $\lambda(t)$ is considered level $J$ inhomogeneous if it is level $J-1$ homogeneous and not level J homogeneous.

We immediately remark that a level $J$ inhomogeneous Poisson process can not be level $j$ homogeneous for all $j \geq J$. In the example represented in Figure 1, the corresponding inhomogeneous Poisson process is level 0 and level 1 homogeneous, but this property does not hold from $J \geq 2$. Also, it is shown in [18] that independently on the localization of $t, \hat{\lambda}^{J}(t)$ simplifies with Haar wavelets into the form $2^{J+1} x_{i}^{t} / T$, where $x_{i}^{t}$ is the event count in the subinterval where $t$ belongs. Therefore we can extract an exact distribution for this estimator under the specific assumption of a Poisson process (homogeneous or not).

Proposition 3.1 With the Haar wavelet basis and for a Poisson process of rate $\lambda(t)$, the rate estimates are distributed as

$$
\hat{\lambda}_{i}^{J} \sim \frac{2^{J+1}}{T} \operatorname{Pois}\left(\mu_{i}\right), \quad 0 \leq i \leq 2^{J+1}-1
$$

where $\mu_{i}=\int_{s_{i}} \lambda(t) \mathrm{d} t$

From this result we can write $E\left\{\hat{\lambda}_{i}^{J}\right\}=\lambda_{i}^{J}=\frac{2^{J+1}}{T} \mu_{i}$, for all $0 \leq i \leq 2^{J+1}-1$. We will use Proposition 3.1 in Section 4 to develop a likelihood ratio test for the $J$-th level homogeneity of a Poisson process.

\section{HOMOGENEITY TEST}

We present here a likelihood ratio test (LRT) for $J$ th-level homogeneity. Although a piecewise analysis has also been carried out in [14], the wavelet approach presented here gives a natural, multiresolution scheme for defining the subdivision of the process. Our testing procedure can be directed towards two different challenges, which are either testing $J^{\text {th }}$-level homogeneity for a Poisson process at some resolution of interest $J$, or testing $J^{t h}$-level inhomogeneity and thus not rejecting $j^{t h}$-level homogeneity for all $0 \leq j<J$.

In Section 3 we have stated that $\hat{\lambda}^{J}(t)$ is a piecewise constant function on well defined intervals $S_{J}$, and that under the assumption of a Poisson process the values it takes on these intervals are independent random variables of known distribution (a scaled Poisson distribution - see Proposition 3.1). Let $\left\{N_{m}, m=1, \ldots, M\right\}$ be a collection of independent realisations of the same Poisson process $N$. Let $\Lambda=\left\{\boldsymbol{\Lambda}_{m}\right\}_{m=1}^{M}$ be the set of $M$ independent random vectors where $\boldsymbol{\Lambda}_{m}$ is the vector of all subinterval estimates of the rate from $N_{m}$, that is $\boldsymbol{\Lambda}_{m}=\left(\hat{\lambda}_{i, m}^{J}\right)_{i=0}^{2^{J+1}-1} \cdot \boldsymbol{\Lambda}_{m}$ is parametrized by the vector $\left(\lambda_{i}^{J}\right)_{i=0}^{2^{J+1}-1}$. We look to test the null hypothesis $H$ which states $N$ is level $J$ homogeneous against alternative hypothesis $K$ which states it is not. The LRT statistic is defined as:

$$
r=\frac{\sup _{\lambda_{c}^{J}>0} \mathcal{L}\left(\Lambda ; \lambda_{c}^{J}, \ldots, \lambda_{c}^{J}\right)}{\sup _{\lambda_{i}^{J}>0} \mathcal{L}\left(\Lambda ; \lambda_{0}^{J}, \ldots, \lambda_{2^{J+1}-1}^{J}\right)}
$$

where $\mathcal{L}\left(\Lambda ; \lambda_{0}^{J}, \ldots, \lambda_{2^{J+1}-1}^{J}\right)$ is the likelihood of the data $\Lambda$ given parameter vector $\left(\lambda_{i}^{J}\right)_{i=0}^{2^{J+1}-1}$. From Proposition 3.1 it can be shown $r$ equals

$$
\exp \left(-\frac{M}{\delta^{J}}\left(2^{J+1} \bar{\lambda}_{c}^{J}-\sum_{i=0}^{2^{J+1}-1} \bar{\lambda}_{i}^{J}\right)\right) \prod_{i=0}^{2^{J+1}-1}\left(\frac{\bar{\lambda}_{c}^{J}}{\bar{\lambda}_{i}^{J}}\right)^{\frac{M}{\delta^{J}} \bar{\lambda}_{i}^{J}}
$$

where $\delta^{J}=2^{J+1} / T, \bar{\lambda}_{i}^{J}=(1 / M) \sum_{m=1}^{M} \hat{\lambda}_{i, m}^{J}$ and $\bar{\lambda}_{c}^{J}=(1 / M) \sum_{m=1}^{M} \hat{\lambda}_{c, m}^{J}$ where $\hat{\lambda}_{c, m}^{J}=\left(1 / 2^{J+1}\right) \sum_{i=0}^{2^{J+1}-1} \hat{\lambda}_{i, m}^{J}$. Statistic $\bar{\lambda}_{c}^{J}$ is the maximum likelihood estimator (MLE) of $\lambda_{c}^{J}$, the constant $J$ th level rate function under the null hypothesis $H$, and $\bar{\lambda}_{i}^{J}$ is the MLE for $\lambda_{i}^{J}\left(i=0, \ldots, 2^{J+1}-1\right)$, under the alternative hypothesis $K$. The expressions of $\bar{\lambda}_{c}^{J}$ and $\bar{\lambda}_{i}^{J}$ let us simplify $r$ into

$$
\prod_{i=0}^{2^{J+1}-1}\left(\frac{\bar{\lambda}_{c}^{J}}{\bar{\lambda}_{i}^{J}}\right)^{\frac{M}{\delta} \bar{\lambda}_{i}^{J}}
$$

The collection of random vectors $\Lambda$ must be obtained by $M$ independent realizations from the same point process. The $M=1$ case would involve only a single realization of the process and consequently dictate that $\hat{\lambda}_{i}^{J}$ be the estimator for $\lambda_{i}^{J}$. In the context of Poisson random variables this case has been dealt with in [19]. However, we must note that in this situation 


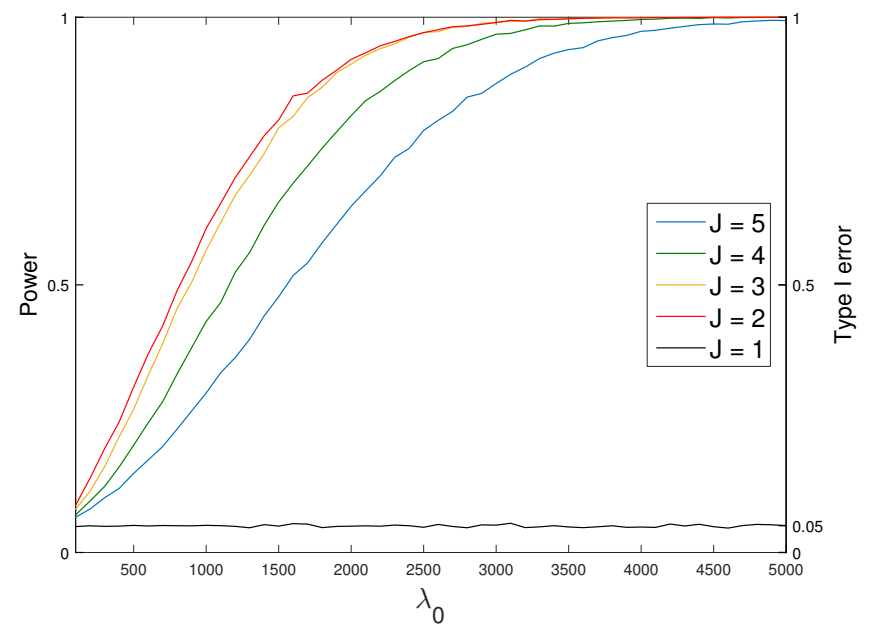

Fig. 2. Type 1 error $(J=1)$ and empirical power $(J \in\{2,3,4,5\})$ for a piecewise triangular rate with $V=1, \xi=1 / 80$, $T=5$.

increasing the number of parameters in the alternative hypothesis, that results from increasing the resolution, brings about consistency issues with the MLE (see [20] for more details). We now derive the following test statistic:

$$
R=-2 \log (r)=-2 \frac{M}{\delta^{J}} \sum_{i=0}^{2^{J+1}-1} \bar{\lambda}_{i}^{J} \log \left(\frac{\bar{\lambda}_{c}^{J}}{\bar{\lambda}_{i}^{J}}\right) .
$$

Let $d_{H}$ be the number of free parameters under the null hypothesis $H$ and let $d_{K}$ be the number of free parameters under the alternative hypothesis $K$, then the null distribution of $R$ is approximately a $\chi^{2}$ distribution with $d_{K}-d_{H}$ degrees of freedom for a large sample size $M$ under some regularity assumptions [21]. In our setting $d_{K}=2^{J+1}$ and $d_{H}=1$ giving $R$ as asymptotically $\chi^{2}$ distribution with $2^{J+1}-1$ degrees of freedom. We reject $J$-th level homogeneity at significance level $\alpha$ if $R>c_{\alpha}$ where $c_{\alpha}$, the critical value, is the upper $100 \alpha \%$ point of the $\chi_{2^{J+1}-1}^{2}$ distribution. This asymptotic result would not have been rigorously applicable if we were restricting ourselves to the $M=1$ case [20].

\section{SIMULATION STUDY}

Here, we demonstrate the LRT for $J$-level homogeneity through simulations. We consider a class of inhomogeneous Poisson processes on a time interval $[0, T]$. These processes share a similar piecewise triangular rate as in Figure 1 . The absolute value of the slope of $\lambda(t)$ is $2^{V+1} \xi \lambda_{0} / T$, where $2^{V}$ is the number of peaks in the rate function. The rate takes values between $\lambda_{0} \frac{1-\xi}{2}$ and $\lambda_{0} \frac{1+\xi}{2}$. By construction, the quantity $\int_{0}^{T} \lambda(t) d t$ does not depend on $V$, the process is level $V$ homogeneous and level $V+1$ inhomogeneous.

We set the significance level of our test at $\alpha=0.05$, with $M=100, V=1, \xi=1 / 80$ and $T=5$. The empirical type 1 error and power of the LRT (over 10000 simulations) at different values of $J$ are shown in Figure 2 as a function of $\lambda_{0}$. We observe that the empirical power converges to 1 when $\lambda_{0} \rightarrow \infty$. The empirical type 1 error, as expected, lies close to the 5\% level (the empirical errors for $J=0$ and $J=1$ are similar). It is noticeable that the power decreases as we increase $J$. This can be explained because when $J$ increases the mass of the null distribution $\chi_{2^{J+1}-1}^{2}$ is displaced to the right, making it harder for the test to distinguish between the null hypothesis and the true state of nature.

\section{CONCLUSION}

In this paper we have focused on a wavelet-based approach to estimate the first-order intensity for point processes on the real line. We have considered a multiresolution reconstruction of the rate function using the Haar wavelet basis. The Haar wavelet basis lends itself to a rigorous statistical analysis and the exact distribution of the estimator of the rate function is presented. This result has been used to design a likelihood ratio test for a new property defined as $J$-th level homogeneity, a multiresolution interpretation of the homogeneity of a Poisson process. Standard asymptotic results for the distribution of the test statistic under the null hypothesis can be applied. A simulation study has demonstrated the test on a suitable example process. 


\section{REFERENCES}

[1] D. R. Brillinger, "Statistical inference for stationary point processes.", in Stochastic Processes and Related Topics. Academic Press, 1975, vol. 1, pp. 55-99.

[2] S. L. Rathbun and N. Cressie, "Asymptotic Properties of Estimators for the Parameters of Spatial Inhomogeneous Poisson Point Processes," Advances in Applied Probability, vol. 26, no. 1, pp. 122-154, 1994.

[3] O. Aalen, "Nonparametric Inference for a Family of Counting Processes," The Annals of Statistics, vol. 6, no. 4, pp. 701-726, 1978.

[4] H. Ramlau-Hansen, "Smoothing counting process intensities by means of kernel functions," The Annals of Statistics, pp. 453-466, 1983.

[5] E. A. K. Cohen, "Multi-wavelet coherence for point processes on the real line," in IEEE International Conference on Acoustics, Speech and Signal Processing (ICASSP). 2014, pp. 2649-2653, IEEE.

[6] D. R. Brillinger, "Some wavelet analyses of point process data," in Conference Record of the Thirty-First Asilomar Conference on Signals, Systems and Computers. Computer Society Press, 1997, vol. 2, pp. 1087-1091, IEEE Comput. Soc.

[7] J. C. S. de Miranda and P. A. Morettin, "Estimation of the intensity of non-homogeneous point processes via wavelets," Annals of the Institute of Statistical Mathematics, vol. 63, no. 6, pp. 1221-1246, 2011.

[8] E. D. Kolaczyk, "Wavelet shrinkage estimation of certain Poisson intensity signals using corrected thresholds," Statistica Sinica, vol. 9, no. 1, pp. 119-135, 1999.

[9] L. J. Bain, M. Engelhardt, and F. T. Wright, "Tests for an Increasing Trend in the Intensity of a Poisson Process: A Power Study," Journal of the American Statistical Association, vol. 80, no. 390, pp. 419-422, 1985.

[10] P. A. W. Lewis, “Some results on tests for Poisson processes," Biometrika, vol. 52, no. 1-2, pp. 67-77, 1965.

[11] E. T. M. Ng and R. J. Cook, "Adjusted Score Tests of Homogeneity for Poisson Processes," Journal of the American Statistical Association, vol. 94, no. 445, pp. 308-319, 1999.

[12] M. Fromont, B. Laurent, and P. Reynaud-Bouret, "Adaptive tests of homogeneity for a Poisson process," Annales de l'I.H.P. Probabilités et statistiques, vol. 47, no. 1, pp. 176-213, 2011.

[13] B. D. Ripley, "Tests of 'randomness' for spatial point patterns," Journal of the Royal Statistical Society, vol. B 41, no. 3, pp. 368-374, 1979.

[14] R. Fierro and A. Tapia, “Testing Homogeneity for Poisson Processes," Revista Colombiana de Estadística, , no. 3, pp. 421-432, 2011.

[15] D. R. Brillinger, P. M. Guttorp, F. P. Schoenberg, A. H. El-shaarawi, and W. W. Piegorsch, "Point processes, temporal," Environmetrics, vol. 3, pp. 1577-1581, 2002.

[16] N. Cressie, Statistics for Spatial Data (revised edition), J. Wiley, 1993.

[17] Y. Meyer, "Wavelets and operators," Cambridge Studies in Advanced Mathematics, vol. 37, 1992.

[18] Y. Taleb and E. A. K. Cohen, "A statistical study of the wavelet estimation of point process intensities," To be submitted.

[19] L. D. Brown and L. H. Zhao, "A new test for the Poisson distribution," The Indian Journal of Statistics, vol. 64, no. Series A, Pt. 3, pp. 1-29, 2002.

[20] C. Feng, H. Wang, and X. M. Tu, "The asymptotic distribution of a likelihood ratio test statistic for the homogeneity of poisson distribution," Sankhyā: The Indian Journal of Statistics, vol. 74-A, no. 2, pp. 263-268, 2012.

[21] S. S. Wilks, "The Large-Sample Distribution of the Likelihood Ratio for Testing Composite Hypotheses," The Annals of Mathematical Statistics, vol. 9, no. 1, pp. 60-62, 1938. 